\title{
Zurich Consensus: German Expert Opinion on the St. Gallen Votes on 15 March 2009 (11th International Conference at St. Gallen: Primary Therapy of Early Breast Cancer)
}

\author{
Participants: Matthias W. Beckmann ${ }^{a}$ Jens-Uwe Blohmer ${ }^{b}$ Serban-Dan Costa ${ }^{c}$ \\ Klaus Diedrich ${ }^{d}$ Ingo Diel ${ }^{e}$ Wolfgang Eiermann ${ }^{f} \quad$ Klaus Friese $^{g}$ Bernd Gerber $^{h}$ \\ Nadia Harbeck $^{i}$ Joern Hilfrich ${ }^{\mathrm{j}}$ Wolfgang Janni ${ }^{\mathrm{k}}$ Fritz Jaenickel Walter Jonat ${ }^{\mathrm{m}}$ \\ Manfred Kaufmann ${ }^{n}$ Marion Kiechle ${ }^{\circ}$ Uwe Koehler $^{p}$ Rolf Kreienberg ${ }^{q}$ \\ Gunter von Minckwitz ${ }^{r}$ Volker Moebus ${ }^{s} \quad$ Ulrike Nitz ${ }^{t} \quad$ Andreas Schneeweiss $^{u}$ \\ Christoph Thomssen ${ }^{v}$ Diethelm Wallwienerw
}

aErlangen University Women's Hospital, 'bt. Gertrauden Hospital, Berlin, 'Magdeburg University Women's Hospital, 'Luebeck University Women's Hospital, e Gynecology and Obstetrics Joint Practice, Mannheim, 'Women's Clinic, Red Cross Hospital, Munich, ${ }^{9}$ Munich University Women's Hospital, 'Rostock University Women's Hospital, 'Cologne University Women's Hospital, 'Eilenriede Hospital, Hanover, ${ }^{k}$ Duesseldorf University Women's Hospital, 'Clinic and Polyclinic for Gynecology, Hamburg-Eppendorf University Hospital, 'miel University Women's Hospital, " Frankfurt/Main University Women's Hospital, ${ }^{\circ}$ Women's Hospital of the Munich Technical Universty, ${ }^{\mathrm{P} C l i n i c}$ for Gynecology and Obstetrics at Klinikum St. Georg gGmbH, Leipzig, ${ }^{9} U l m$ University Women's Hospital, 'German Breast Group, Neu-Isenburg, 'Women's Clinic at Hoechst Hospital, Frankfurt/Main, 'Bethesda Lutheran Hospital, Moenchengladbach, "University Women's Hospital and National Center for Tumor Diseases, Heidelberg, vUniversity Hospital and Polyclinic for Gynecology, Halle (Saale), wTuebingen University Women's Hospital, Germany

\section{Summary}

A German working group of 23 breast cancer experts discussed the results from the vote at this year's St. Gallen Consensus Conference on Primary Therapy for Early Breast Cancer (March 11-14, 2009) and came up with some concrete recommendations for day-to-day therapeutic decisions in Germany. Due the fact that the concept of the St. Gallen Consensus Conference merely allows for a minimal consensus, the objective of the working group was to provide practice-related recommendations for day-to-day clinical decisions in Germany. One area of emphasis at St. Gallen was tumor biology as a starting point for reaching individual therapeutic decisions. Intensive discussion was necessary with respect to the clinical relevance of predictive and prognostic factors. A new addition to the area of systemic therapy was a first-ever discussion of the adjuvant administration of bisphosponates and the fact that therapy with trastuzumab in HER2 overexpressing breast cancer has been defined as the standard for neoadjuvant therapy. The value of taxanes as a component of (neo)adjuvant chemotherapy as well as the value of aromatase inhibitors for the endocrine adjuvant treatment of postmenopausal patients were affirmed.

\section{Zusammenfassung}

Eine deutsche Arbeitsgruppe aus 23 Brustkrebsexperten hat die Abstimmungsergebnisse der diesjährigen St.-Gallen-Konsensuskonferenz zur primären Behandlung des Mammakarzinoms (11.-14. März 2009) kommentiert und für den Therapiealltag in Deutschland konkretisiert. Vor dem Hintergrund, dass das Konzept der St.-Gallen-Konsensuskonferenz nur einen Minimalkonsensus erlaubt, war es das Ziel der Arbeitsgruppe, praxisrelevante Empfehlungen für den klinischen Alltag in Deutschland zu geben. Ein inhaltlicher Schwerpunkt war in St. Gallen die Tumorbiologie als Ausgangspunkt für die individuelle Therapieentscheidung. Intensiver Diskussionsbedarf bestand bei der klinischen Relevanz prädiktiver und prognostischer Faktoren. Neu im Bereich der systemischen Therapie war, dass erstmals über die adjuvante Gabe von Bisphosphonaten diskutiert wurde und dass die Therapie mit Trastuzumab bei HER2-Überexpression jetzt auch für die neoadjuvante Therapie als Standard definiert wurde. Der Stellenwert der Taxane als Bestandteil der (neo)adjuvanten Chemotherapie sowie der Aromatasehemmer für die endokrine adjuvante Behandlung der postmenopausalen Patientin wurden bestätigt.

\begin{tabular}{ll}
\hline KARGER & ( 2009 S. Karger GmbH, Freiburg \\
$\begin{array}{l}\text { Fax +497614520714 } \\
\text { Information@Karger.de } \\
\text { www.karger.com }\end{array}$ & $\begin{array}{l}\text { Accessible online at: } \\
\text { www.karger.com/brc }\end{array}$
\end{tabular}




\section{Introduction}

This year's 11th International St. Gallen Consensus Conference on the Diagnosis and Treatment of Primary Breast Cancer (March 11-14, 2009) has worldwide significance. This year's panel at St. Gallen was made up of 43 experts from 17 countries, 3 of whom were representatives from Germany. Due to the fact that the recommendations are based on the vote of the representatives from different countries with often very diverse health systems and resources, it was only possible to achieve a minimal consensus. As a result, a German working group of 23 breast cancer experts discussed the voting results from St. Gallen as they relate to the therapy situation in Germany with the objective of formulating some concrete recommendations for day-to-day clinical decision-making in Germany.

The objective of this year's St. Gallen Consensus Conference was to vote on controversial issues and discuss them based on international findings. The results from controlled clinical studies form the basis for an evidence-based therapy. However, it is always necessary to re-evaluate the significance of this 'evidence' for therapeutic decision-making in individual cases - while taking the individual circumstances into consideration.

With this objective in mind, the focus at St. Gallen was on 10 topic areas. In addition to the classic areas of surgery, radiation, endocrine and cytostatic therapy, other areas included the application of targeted substances, as well as the value of predictive and prognostic factors. New areas of discussion included the adjuvant use of bisphosphonates, fertility preservation under oncological treatment, as well as male breast cancer. The panelists responded to the questions submitted for a vote by responding with 'yes' (agree), 'no' (disagree), or 'abstain/don't know'.

\section{Prognostic and Predictive Factors}

The clinical significance of diverse predictive and prognostic factors as markers for certain biological characteristics of tumors was a topic of intensive discussion at St. Gallen. A vote was held on the significance of grading, hormone receptor status and HER2 status, as well as the clinical value of Ki-67 and the use of new molecular tests.

\section{Grading}

Grading was affirmed as a predictive factor for the use of a chemotherapy regimen (G3). Discussion also included the issue of whether so-called 'genomic grading' or other gene expression analyses using molecular testing systems provide a meaningful supplement to histological grading. While the majority of the panelists at St. Gallen agreed that in fact they do, the German experts currently reject the general clinical use of these new testing systems. Their criticism is based on the fact that to date only retrospective data are available on the value of such analytical methods, access to these data is still limited, and these complex procedures have yet to be completely standardized.

\section{Estrogen and Progesterone Receptor}

The panel participants at St. Gallen voted to quantify the estrogen receptor expression based on the percentage of positive cells. The majority accepted a value of $>50 \%$ dye-stained cells as the cut-off value for a new category of highly endocrine-sensitive tumors. The German working group agreed that a highly endocrine-sensitive tumor can be assumed at a value of $>50 \%$ positive cells, but that this does not constitute an exclusionary criterion. To date there is no known evidencebased cut-off value for the authoritative definition of an endocrine-sensitive or highly sensitive breast carcinoma. Thus the German working group pointed out that the described situation (cut-off $>50 \%$ ) is merely one example of a highly endocrine-sensitive tumor and that other constellations do not rule out an endocrine sensitivity. Tumors can be less deeply dye-stained but still respond to endocrine therapy.

The majority of the panelists accepted a value of $<10 \%$ positive cells as a cut-off for an endocrine-insensitive tumor. Again the German working group disagreed: a tumor is not certain to be endocrine-insensitive except in cases where no positive cells can be detected. Unlike the other panelists at St. Gallen, the German experts urged the use of additional scores that take into consideration the intensity of cell staining as well as the number of dye-stained cells, in order to quantify endocrine sensitivity. It was also asserted that a Remmele score $\geq 9$, for example, or an Allred score $\geq 7$ is indicative of a tumor with high endocrine sensitivity. The greatest importance and priority must be placed on adequate quality assurance in determining hormone receptor expression.

The German experts agreed with the St. Gallen panelists that the progesterone receptor $(\mathrm{PgR})$ has a prognostic value, but does not provide any predictive value for the use of tamoxifen or an aromatase inhibitor (AI). The German working group further asserted that the $\mathrm{PgR}$ is merely a general predictor for the response to an endocrine therapy. The study data on postmenopausal patients show a comparable relative risk reduction independent of the $\mathrm{PgR}$ expression under AIs as compared to tamoxifen.

\section{HER2 Expression}

HER2 overexpression is the precondition for treatment with trastuzumab. The discussion involved determining at what immunohistochemical (IHC) cut-off value therapy with the monoclonal antibody is indicated. The German experts concurred with the St. Gallen decision to raise the cut-off value from $10 \%$ - as defined in the clinical studies - to $30 \%$ positive cells (cf. ASCO recommendations: Wolfe et al., JCO 2007). This is equivalent to IHC3 $+(\geq 30 \%$ positive $)$. In cases of doubt, a positive FISH test is required in order to initiate treatment with trastuzumab. 
A significant majority of the panelists at St. Gallen considered HER2 overexpression a predictor for neoadjuvant use of anti-HER2 medication. In accord with the German experts, St. Gallen recommended the additional use of trastuzumab with HER2 overexpression not only for adjuvant, but also for neoadjuvant chemotherapy. In the GBG/AGO meta-analysis of neoadjuvant chemotherapy (by Minckwitz et al., SABCS 2008, abstract) the additional administration of trastuzumab for chemotherapy was an independent predictor for the achievement of a pathological complete remission. It was also agreed that HER2 overexpression is a predictor for the response to (neo)adjuvant chemotherapy in general. Conversely, HER2 overexpression is not a predictor for the response to a certain endocrine therapy. However, the higher risk associated with HER2 overexpression - similar to a negative $\mathrm{PgR}$ - justifies the primary use of an AI.

\section{Proliferation Marker Ki-67}

The use of the proliferation marker Ki-67 as a prognostic and/or predictive factor was discussed intensively at St. Gallen. The majority of panelists considered Ki-67 a prognostic factor. The vote on the predictive value of $\mathrm{Ki}-67$ was controversial. The German experts currently view Ki-67 as neither a valid prognostic nor a predictive factor because there are no supporting data available from prospective studies. The German working group made reference to unresolved issues in the determination of Ki-67, e.g. a lack of standardization, the lack of a clearly defined cut-off value, and the fact that Ki67 has not been confirmed to be an independent factor in all clinical studies. According to the German working group, the routine determination of Ki-67 alone currently has no clinical relevance for therapeutic decision-making.

\section{uPA/PAI-1 Determination}

The routine determination of UPA/PAI-1 as a prognostic factor was rejected by the majority of panelists at St. Gallen. The German experts did not concur. They view uPA/PAI-1 as a valid, standardized, and evidence-based method whose clinical relevance for node-negative breast cancer is supported by prospective data. uPA/PAI-1 is a clinically relevant factor for the decision for or against adjuvant chemotherapy for tumors with a clinically intermediate risk profile.

\section{Gene Expression Analyses}

The clinical significance of molecular gene expression analyses was discussed at St. Gallen based on the example of Oncotype DX $^{\circledR}$ (Genomic Health, Inc., Redwood City, CA, USA) and Mammaprint ${ }^{\circledR}$ (Agendia, Huntington Beach, CA, USA). The panel was basically in agreement that the use of currently available molecular tests in addition to clinical and histopathological parameters, in other words not as the sole instrument, can be useful, e.g. in patients with hormone-sensitive breast cancer, for whom it is unclear whether adjuvant chemotherapy is indicated, following the consideration of histopathological criteria. In cases of doubt, however, these patients should be treated within corresponding clinical studies, such as the MINDACT study. The German experts currently see no value in molecular tools, such as gene expression analyses, outside of clinical studies. Their main argument is that only retrospective data are available to date.

\section{CYP2D6 Determination}

As posited at St. Gallen, the German working group also does not currently view CYP2D6 as a general predictor for endocrine therapy decision-making in postmenopausal patients and for the question of whether they should be treated with tamoxifen or an AI. As a result, they - as well as the majority of the panelists at St. Gallen - reject a routine CYP2D6 test in patients who are supposed to receive tamoxifen. The German experts pointed out that currently there are no prospective data available to justify a general CYP2D6 test. The percentage of patients who metabolize tamoxifen very poorly ('poor metabolizers') is only about $6-7 \%$ in Germany. The clinical consequences are unclear for the nearly $20 \%$ of 'intermediate metabolizers'. If a valid CYP2D6 test is already available from a standardized laboratory, then the patient should be notified about the findings and receive the necessary advice. However, an active recommendation for a CYP2D6 test is currently not indicated.

\section{Local Breast Cancer Therapy}

\section{Axillary Dissection, Additional Resection}

At St. Gallen the votes on local therapy for primary early breast cancer focused on axillary dissection, additional resection, and radiation therapy. In each case, a significant majority of the St. Gallen panel considered the sentinel lymph node biopsy (SLNB) the standard in cases of invasive breast cancer with clinically normal axilla (cN0) - with the exception of inflammatory breast cancer (T4d). Axillary dissection should be performed in cases where micrometastases are detected in the sentinel SLNB. Where necessary, axillary dissection may be dispensed with only in select patients with low risk $(\mathrm{T} \leq 1 \mathrm{~cm}$, N0, G1, max. 1 affected SLN out of at least 5 removed SLN, L0, V0 ER/PgR-positive, HER2-negative).

Likewise, considerable majorities at St. Gallen were in favor of additional resection. It was affirmed that additional resection is necessary in cases of an invasive carcinoma or DCIS (ductal carcinoma in situ) with positive margins (R1). Conversely, the majority agreed that additional resection is not necessary in cases of an accompanying LCIS (lobular carcinoma in situ). The German experts concurred with the vote for the accompanying LCIS, but they pointed out that the situation is totally different in cases of a solitary, dedifferentiated (plenomorphic) LCIS. Discussions are currently ongoing to reclassify LCIS and to differentiate the types more clearly - with corresponding surgical implications. 
Similarly controversial at St. Gallen was the issue discussed by the German experts as to whether a resection margin of $2 \mathrm{~mm}$ is sufficient to forego an additional resection with the presence of a solitary DCIS. This question was put to a vote and verbally approved at St. Gallen. The discussion was based on the data from a current meta-analysis with a total of over 4,000 patients, according to which a $2-\mathrm{mm}$ resection margin is sufficient in cases of DCIS (Dunne et al., JCO 2009).

This question and vote were in clear opposition to the 2009 recommendations of the 'AGO Kommission Mamma' and the S3 guideline of the German Cancer Society, which recommend a resection margin $\geq 5-10 \mathrm{~mm}$ in cases of solitary DCIS. The current data from the meta-analysis could result in an important change in strategy. The majority of German experts was of the opinion that, depending on the size of the tumor, free resection margins with a minimum distance of $>2$ $\mathrm{mm}$ are necessary in the case of a solitary DCIS (exceptions: skin, pectoral fascia). However, the German working group also pointed out that these changes still need to be discussed before the German guidelines can be modified.

\section{Radiation Therapy}

A significant majority of the panelists at St. Gallen affirmed radiation therapy as the post-operative standard for patients with DCIS. About two-thirds of the panelists voted in approval of foregoing radiation in cases of older patients ( $>75$ years of age) and those with low-grade DCIS (low risk). The German working group voted in favor in both votes. They did present one condition: an individual risk/benefit analysis must be performed prior to foregoing radiation therapy in older women and/or in low-risk situations.

The majority of panelists approved accelerated whole breast radiation therapy (WBRT) as the acceptable option for patients over 60 years of age with invasive cancer. The German working group concurred. Conversely, the vote on the value of intraoperative partial breast radiation therapy (PBRT) was intensively debated. The majority of the panelists at St. Gallen classified PBRT as an experimental procedure. The German working group concurred with the majority decision. A minority of German experts pointed out, however, that PBRT represents an option for certain day-to-day clinical situations, within the framework of clinical studies wherever possible, e.g. for low-risk patients (T1, N0, L0, V0, G1, ER/ PgR-positive, unicentric, postmenopausal, HER2-negative, no EICs). Essentially the German working group referred to the ASCO and ASTRO recommendations, which clearly define when PBRT can be indicated, e.g. instead of boost radiation, and when it is contraindicated.

There was consensus on the St. Gallen vote concerning the indication for radiation therapy in breast cancer patients following mastectomy. It is the standard for patients with at least four affected lymph nodes and an option for patients with 1-3 affected lymph nodes - especially for younger women $(<50$ years of age) and those with unfavorable prognostic factors.
The EBCTCG (Early Breast Cancer Trialist Collaborative Group) meta-analysis found a survival benefit for patients with 1-3 affected lymph nodes after 15 years (Peto et al., NEJM 2005). According to a subgroup analysis, the survival benefit applied especially to patients with 2-3 affected lymph nodes. The German group of experts criticized the fact that the systemic therapy included in the Peto analysis typically did not conform to today's standards.

For years follow-up radiation has been the standard following breast-conserving operations. According to the vote at St. Gallen, this is also valid for patients with minor, hormone-sensitive breast cancer (T1N0), who receive endocrine treatment, as well as for postmenopausal patients with hormone-sensitive breast cancer and a life expectancy of more than 10 years. The German experts concurred with the St. Gallen vote. They also pointed out that the local recurrence rate in older patients was considerably reduced through the use of radiation as well ( 1 vs. $7 \%$ after 10 years). The German working group emphasized that endocrine therapy cannot replace radiation therapy following breast-conserving operations even for older patients (>70 years of age, ER-positive), following an unclear vote on the issue at St. Gallen (51 vs. $46 \%$ ).

\section{Endocrine Therapy}

\section{Premenopausal Patients}

For premenopausal patients with hormone-sensitive primary breast cancer both the exclusive treatment with tamoxifen (preferred), as well as the combination of tamoxifen plus ovarian function suppression (OFS), is one possible standard. The German working group concurred with the majority of the panelists at St. Gallen on both of these options. However, the German experts were of the more restricted opinion that the combination treatment with tamoxifen plus OFS (LHRH analog) is only an additional option in individual cases, e.g. for patients under 40 years of age. Since premenopausal patients with increased risk of recurrence usually receive chemotherapy in addition to endocrine treatment, the scope of application for LHRH analogs plus tamoxifen is relatively narrow. The German working group warned of overtreatment with LHRH analogs. Treatment with OFS plus tamoxifen can reduce the risk of recurrence in women under 40 years of age and with reinstated ovarian function following chemotherapy. Whether and/or which patients actually benefit from the additional administration of the LHRH analog is currently being investigated by the SOFT and the TEXT studies, among others.

From the German standpoint, OFS alone in otherwise healthy women is the standard. At least $40 \%$ of the panelists at St. Gallen viewed OFS alone as a standard approach. The German working group pointed out that OFS alone only represents a therapy option in individual cases, e.g. in women with a medical contraindication to tamoxifen. With respect to the duration of therapy, the German working group added 
that tamoxifen can be used for 5 years and LHRH analogs can be used for 2-5 years, depending on the therapeutic concept. Due to time limitations, the St. Gallen conference did not vote on the use of AIs in premenopausal patients with hormonesensitive breast cancer. Here the German working group added that AIs alone are not typically indicated in premenopausal patients. They are only an option in cases of medical contraindications to tamoxifen - and then only in combination with LHRH analogs.

\section{Postmenopausal Patients}

The discussion focused on the value of tamoxifen versus AIs in the treatment of postmenopausal patients with hormonesensitive breast cancer. Over the course of the voting, the majority of St. Gallen panelists considered exclusive treatment with tamoxifen as well as treatment with an AI possible standard approaches for postmenopausal patients. Here the German working group specified that from the German standpoint, exclusive treatment with tamoxifen is only an option for postmenopausal patients with hormone receptor positive breast cancer and very low risk of recurrence or advanced age. Moreover, tamoxifen is the standard for the prevention of contralateral breast cancer. In all other cases AI is a sturdy component of adjuvant endocrine therapy for postmenopausal patients with hormone-sensitive breast cancer.

From the German standpoint, if there is an indication for AI, then it should initially be used in accordance with the individual risk profile of the patient or after treatment for 2 years with tamoxifen. The greater the recurrence risk is (stage, especially nodal status, tumor size, HER2 status, PgR status, proliferation index), the greater the preference for the initial use of AI from the German standpoint. If the AI has been used up front, then there is the option to switch to tamoxifen after 2 years, e.g. in patients with AI-related adverse reactions. As a result, the duration of AI therapy will vary and usually lasts 2-5 years, depending on the therapeutic concept. For an indication for treatment with an AI, the panelists at St. Gallen had a preference for beginning a therapy with an AI, which included both the 5-year up front therapy, as well as the switch to tamoxifen after 2 years (inverse sequence). Only $15 \%$ of the panelists voted for the planned staggered use of the AI after 2-3 years of therapy with tamoxifen.

HER2 Overexpression with Hormone-Sensitive Breast Cancer The majority opinion among the panelists at St. Gallen was that a HER2 overexpression with hormone-sensitive breast cancer does not represent a preferred indication for an AI. The German working group found it important to point out that the HER2 status is merely a predictor for the response to trastuzumab, but not a predictive factor for the use of AI versus tamoxifen. Patients with hormone-sensitive HER2overexpressing breast cancer usually respond more poorly to an endocrine therapy than patients without HER2 overexpression. This is equally true for both tamoxifen and AI. The relatively better effect of the AIs as compared to tamoxifen depends on the HER2-expression; the absolute benefit alone is greater for the AI in cases with HER2 overexpression. Thus the German working group agreed that treatment with an AI is recommended due to the increased risk of recurrence associated with HER2 overexpression in postmenopausal patients.

Endocrine adverse reactions under adjuvant endocrine therapy are not an indication of an especially good therapeutic response in patients. The German experts agreed here with the vote at St. Gallen without reservations. They were also in agreement with the vote at St. Gallen concerning the question of the options for conserving ovarian function in patients during chemotherapy. To date a standard method has yet to be defined in this regard. At present, ovarian stimulation and IVF (in vitro fertilization) are the only established methods for effective cryopreservation.

\section{Male Breast Cancer}

In rare cases men can also develop breast cancer (approximately 400-500 new cases per year in Germany). The endocrine therapy standard here is treatment with tamoxifen. AI is currently not indicated for men. The German working group added that data collection on male breast cancer should be more centralized and greater effort should be made to conduct clinical studies on the disease.

\section{Adjuvant Chemotherapy}

\section{Hormone-Sensitive Breast Cancer without HER2 \\ Overexpression}

The focus here was on the use of adjuvant chemotherapy in patients with highly endocrine-sensitive breast cancer. A majority on the St. Gallen panel answered 'yes' to the question of whether a highly endocrine-sensitive tumor with HER2 overexpression responds less well to adjuvant chemotherapy. The additional administration of adjuvant chemotherapy was thus considered to be 'less useful' for clinical use in hormone receptor positive patients without HER2 overexpression. The German working group interpreted these questions differently than the consensus panel. Both the Oxford meta-analysis (EBCTCG, Lancet 2005) as well as a current meta-analysis with docetaxel-containing regimens (by Andre et al., JCO 2008) demonstrate that patients with a chemotherapy indication - regardless of age and hormone receptor status - benefited from an adjuvant chemotherapy with a similar, relative risk reduction. This just means that patients with low risk of recurrence have a slighter absolute benefit from the adjuvant chemotherapy.

Based on the regimen used, controlled clinical studies on paclitaxel administered for 3 weeks showed that the effect was somewhat slighter in patients with hormone receptor positive breast cancer than in hormone receptor negative women 
Table 1. Adjuvant chemotherapy - standard regimen according to Zurich Consensus 2009 (no standard regimen was designated at St. Gallen)

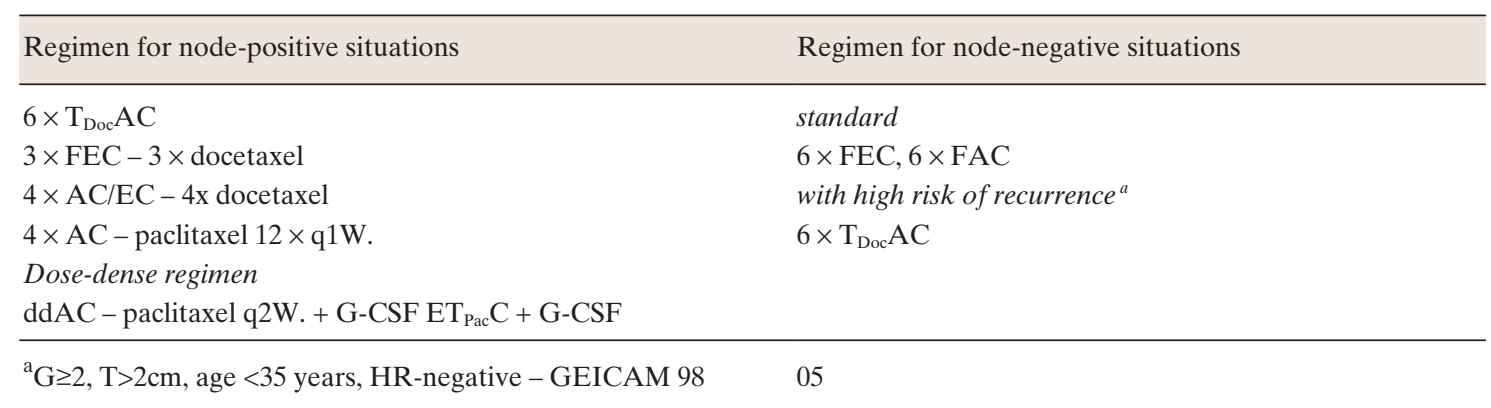

(Hayes et al., NEJM 2007). However, this might be related with an underdosing of the substance. The three-week administration of docetaxel and/or the weekly administration of paclitaxel are similarly effective. In the BCIRG 001 study patients with hormone receptor positive breast cancer benefited just as much from the treatment with the triple $\mathrm{T}_{\text {Doc }} \mathrm{AC}$ combination as the hormone receptor negative patients (Martin et al., NEJM 2005).

\section{Standard Regimen Depending on HER2 Status}

The majority of the St. Gallen panel rejected the notion that there are different standard chemotherapy regimens for patients with early breast cancer depending on the HER2 and/or hormone receptor status of the tumor. This was also the case for 'triple negative' breast cancers (ER-, PgR-, HER2-). The German working group concurred with this opinion.

Anthracycline- and taxane-containing regimens are the standard for the adjuvant treatment of patients with HER2positive and HER2-negative breast cancer. For each type there are several regimens to choose from - depending on nodal status and/or the individual risk constellation. Patients with HER2 overexpression require trastuzumab in addition to chemotherapy. Trastuzumab can be used sequentially or simultaneously with the chemotherapy.

Both of the anthracycline-containing, triple combination FEC and/or FAC regimens, 6 cycles each, are the standard for patients without lymph node involvement (N0) and an indication for chemotherapy. In direct and indirect comparisons the triple combinations are superior to the $\mathrm{CMF}$ regimen (EBCTCG, Lancet 2005). This could not be proven for the anthracycline-containing double combinations even with more than four therapy cycles. In cases of increased risk of recurrence $(\mathrm{G} \geq 2, \mathrm{~T}>2 \mathrm{~cm}$, age $<35$ years, HR-negative, according to GEICAM 9805) the German working group recommends the use of 6 cycles of $\mathrm{T}_{\mathrm{Doc}} \mathrm{AC}$ (docetaxel, doxorubicin, cyclophosphamide) (table 1 ). The $\mathrm{T}_{\text {Doc }} \mathrm{AC}$ regimen in the randomized phase III study achieved a significant advantage in disease-free survival time for patients without lymph node involvement compared with 6 cycles of FAC (Martin et al., ASCO 2008, abstract).

For patients with lymph node involvement, the German working group recommends, as in the AGO guidelines, pri- marily the following anthracycline-/taxane-containing regimens: $6 \times \mathrm{T}_{\text {Doc }}$ AC, $3 \times$ FEC- $3 \times$ Doc, $4 \times$ AC- $12 \times$ Pac/q1W, 4 $\times$ AC- $4 \times$ Doc, as well as $4 \times$ EC- $4 \times$ Doc (table 1 ).

\section{The Role of Dose-Dense Chemotherapy}

Adjuvant treatment with dose-dense chemotherapy was also considered an option at St. Gallen. The German experts concurred with this opinion, adding: dose-dense chemotherapy is an option for risk patients with extensive lymph node involvement at experienced centers. Established regimens include the ETC regimen (epirubicin, paclitaxel, cyclophosphamide) as well as dose-dense administration of AC-paclitaxel, every two weeks. In both regimens the patients also require G-CSF support (Moebus et al., SABCS 2006, abstract; Citron et al., JCO 2003).

\section{Value of Anthracyclines in the Adjuvant Situation}

If there are contraindications against anthracyclines, e.g. due to a preexisting cardiac disease or risk constellation, the $\mathrm{T}_{\text {Doc- }}$ $\mathrm{CbH}$ regimen (docetaxel, carboplatin, trastuzumab) may be an alternative for cases of HER2 overexpression - assuming that there is no resulting contraindication for trastuzumab. The BCIRG 006 study demonstrated the efficacy of $\mathrm{T}_{\mathrm{Doc}} \mathrm{CbH}$ as an anthracycline-free, trastuzumab-containing regimen (Slamon et al., SABCS 2006, abstract). However, it should be noted that only patients without cardiac impairment were enrolled in that study.

The double combination $\mathrm{T}_{\mathrm{Doc}} \mathrm{C}$ (4 cycles of docetaxel/cyclophosphamide) was discussed as the standard option for patients without HER2 overexpression and anthracycline contraindication. There was no majority agreement on this at St. Gallen. The German working group considers the 4 cycles of AC (doxorubicin, cyclophosphamide) in the direct phase III study comparison (Jones et al., JCO 2009). The $\mathrm{T}_{\mathrm{Doc}} \mathrm{C}$ combination with 6 cycles is currently being further validated in clinical studies due to the fact that the therapy duration of 4 cycles (12 weeks), which has been studied in clinical studies, appears to be too short in neoadjuvant therapy, according to the meta-analysis (Minckwitz et al., SABCS 2008, abstract). Moreover, TC has not yet been compared with any of the current standard combinations with three substances. 


\section{Study Participation Encouraged}

Participation in randomized clinical studies was essentially encouraged. Different adjuvant therapy optimization studies are currently being conducted in Germany: the use of the anthracycline-free $6 \times \mathrm{T}_{\text {Doc }} \mathrm{C}$ regimen (docetaxel/cyclophosphamide) in patients without HER2 overexpression is being compared with EC-docetaxel in the Plan B study and with FEC-docetaxel in the SUCCESS C study. The dose-dense/tailored concept is being further validated in the PANTHER study in patients with lymph node involvement with tolerability-related adjusted doses of EC-Doc versus FEC-Doc. The use of $\mathrm{T}_{\text {Doc }} \mathrm{CbH}$ is being studied in patients with HER2-overexpressing breast cancer plus/minus bevacizumab.

\section{Neoadjuvant Chemotherapy}

The neoadjuvant therapeutic concept has become well established in the treatment of early breast cancer. This was clearly expressed at St. Gallen. The German experts also pointed out that an especially high value is placed on neoadjuvant therapy in Germany. The therapeutic approach was further developed and validated in Germany within the framework of large prospective clinical studies. The structure of the healthcare system in Germany makes it easier to use this form of therapy because overall patient care is primarily in the hands of the gynecologist.

\section{Indication for Neoadjuvant Therapy}

Neoadjuvant chemotherapy basically comes into question if adjuvant chemotherapy is also indicated. The same regimens for adjuvant therapies can be used in neoadjuvant therapies as well. This was affirmed by the great majority of the panelists at St. Gallen (97\%). According to the German experts, the main objectives of neoadjuvant therapy are to increase the rate of breast-conserving operations (BCT) and to improve operative options. They emphasized that not only does neoadjuvant therapy enable operability per se, but it also makes the breast-conserving operation easier to perform because less tissue has to be removed when the response is good ('new limits'). It is beyond dispute that the operation following neoadjuvant chemotherapy requires an experienced surgeon.

Another benefit of neoadjuvant therapy is in vivo chemosensitivity testing. It enables the early individual detection of a therapeutic response, thereby also making it easier to define new predictive factors. The achievement of a pathological complete remission ( $\mathrm{pCR}$ ) is a valid surrogate marker for long-term survival.

Similar to the situation with adjuvant therapy, neoadjuvant chemotherapy is less effective in patients with a low risk profile, e.g. a tumor with a low proliferation rate. This was affirmed by $59 \%$ of the panelists at St. Gallen. A low proliferation rate is to be assumed, for example, in cases of a highly differentiated tumor, a clearly hormone-sensitive breast car- cinoma, a low Ki-67 value, and an invasive lobular tumor as well, with some reservations. $76 \%$ of the St. Gallen panelists rejected the use of a neoadjuvant therapy in these patients. The German working group pointed out that in this situation even adjuvant chemotherapy is only presumably indicated and it is difficult to define a low-proliferating tumor; thus it is necessary to consider whether or not a neoadjuvant therapy is useful in each individual case.

\section{Substance Selection for Neoadjuvant Chemotherapy}

The German experts concurred with the clear majority of the panelists at St. Gallen concerning the substance selection for neoadjuvant chemotherapy. Anthracycline- and taxane-containing regimens are the standard. With HER2 overexpression an anti-HER2 medication with trastuzumab is indicated in addition to neoadjuvant chemotherapy. This was affirmed by $90 \%$ of the panelists at St. Gallen.

At St. Gallen there was no vote on the duration of therapy for neoadjuvant chemotherapy. Based on the large metaanalysis of GBG/AGO (by Minckwitz et al., SABCS 2008, abstract) the German working group recommended a treatment duration of at least 18 weeks. The German working group pointed out that an additional postoperative chemotherapy regimen following previous neoadjuvant chemotherapy outside of clinical studies is not indicated and the operation following neoadjuvant chemotherapy should be performed within the new tumor margins. Moreover, a breast-conserving operation following neoadjuvant chemotherapy is not indicated in cases of extensive microcalcification, inoperable breast cancer and/or a T4 tumor with no therapeutic response, as well as in all cases when post-operative radiation following a breast-conserving operation is not possible.

\section{Neoadjuvant Endocrine Therapy}

Neoadjuvant endocrine therapy is essentially an option in cases of highly hormone-sensitive tumors. $90 \%$ of the panelists at St. Gallen agreed; the German experts concurred. The duration of therapy still remains unclear. This was also evident in the voting results: $20 \%$ voted for $1-4$ months, $43 \%$ for $4-8$ months and $37 \%$ until the best therapeutic response is achieved. The German working group, in accord with the voting results at St. Gallen, favored a duration of therapy of at least 4-8 months.

\section{Bisphosphonates, Targeted Substances, Lifestyle}

\section{Adjuvant Use of Bisphosphonates}

At St. Gallen a vote was held for the first time on the use of bisphosphonates in early primary breast cancer. However, the question put to a vote concerning adjuvant use in addition to endocrine therapy focused on the bisphosphonate zoledronate (every 6 months, i.v.). The panelists at St. Gallen rejected the general adjuvant use of zoledronate in addition to adjuvant 
endocrine therapy regardless of the menopausal status of the patient.

The German working group made additional remarks here by adding that patients can benefit from the use of a bisphosphonate (zoledronate, clodronate) as a component of an adjuvant tumor therapy regardless of menopausal status and the remaining adjuvant therapy. Current clinical findings have shown that additional adjuvant treatment with a bisphosphonate reduces the rate of recurrence, which also showed up as a lower mortality rate in individual studies. Serious adverse reactions or complications were rare in the adjuvant therapy studies.

The German working group also pointed out that bisphosphonates can reduce the risk of tumor therapy-induced osteopenia and/or osteoporosis (TTIO) and the risk of fracture in patients with manifest osteoporosis. As a result, the German experts see an indication for biphosphonates not only for the treatment but also for the prevention of TTIO, following an adequate assessment of the risks - despite the lack of an authorization for such an indication.

\section{Targeted Therapy}

The St. Gallen panel affirmed that patients with HER2 overexpressing early breast cancer should receive trastuzumab in addition to (neo)adjuvant chemotherapy. The German experts concurred with this opinion without reservations. A differentiation of the chemotherapy is not recommended. The panelists at St. Gallen, as well as the German experts, are in agreement that trastuzumab is only indicated together with an indication for chemotherapy. The panelists at St. Gallen viewed the simultaneous and sequential administration of trastuzumab as equally effective options. The majority of the German experts concurred with this opinion.

However, a minority of the German experts favored the simultaneous administration of trastuzumab for chemotherapy, while pointing out inter alia that the clinical findings here show slight benefits. This is comprehensible insofar as the preclinically observed synergistic effects between chemotherapy and trastuzumab can best be put to use with simultaneous administration. It should be noted that there is a higher cardiac risk associated with the simultaneous administration of trastuzumab and anthracyclines, which can be avoided by using $\mathrm{T}_{\text {Doc }} \mathrm{CbH}$.

The majority among the German working group concurred that currently there is not enough evidence in support of simultaneous or sequential administration of trastuzumab plus chemotherapy. The therapeutic decision should be made on an individual basis - also in relation to the chemotherapy used or the planned studies. It is vital that patients with HER2overexpressing breast cancer receive trastuzumab in addition to chemotherapy. The duration of the trastuzumab therapy is one year. Currently there is no indication for the adjuvant use of other targeted therapies outside of clinical studies. This is also true for bevacizumab. The St. Gallen panel and the German experts concurred that bevacizumab is currently not indicated in adjuvant therapy situations. Participation in clinical studies, e.g. the BETH study, is recommended.

\section{Lifestyle and Complementary Medicine}

Issues of patient lifestyle and its possible effects on therapeutic success, as well as the role of complementary medicine, were not discussed at St. Gallen. The German working group added its own observation that lifestyle, especially the combination of physical activity and well-balanced, healthy nutrition can have a positive influence on the prognosis for patients with breast cancer. This is especially applicable to the adjuvant therapy situation. The influence of lifestyle on the prognosis is currently being studied prospectively in the SUCCESS C study. The German working group urged that special care should be taken with complementary and especially socalled alternative medications, as well as extreme diets, which can have a negative effect on the chances for recovery. 